\title{
COUNTEREXAMPLES INVOLVING GROWTH SERIES AND EULER CHARACTERISTICS
}

\author{
WALTER PARRY
}

(Communicated by Bhama Srinivasan)

\begin{abstract}
This note presents examples for which the value of a finitely generated group's growth series at 1 is not the reciprocal of the group's Euler characteristic.
\end{abstract}

Let $G$ be a group generated by the finite set $S$. Given an integer $n \geq 0$, the $n$-ball for the $S$-word metric on $G$ is

$$
B(n)=\left\{g_{1} \cdots g_{n}: g_{i} \in S \cup S^{-1} \cup\{1\} \text { for } i=1, \ldots, n\right\} .
$$

Let $b_{n}$ be the order of $B(n)$ for $n \geq 0$. For $n \geq 1$ let $a_{n}=b_{n}-b_{n-1}$, the order of the $n$-sphere, and set $a_{0}=1$. The growth series for the pair $(G, S)$ is defined by

$$
f(X)=f(G, S, X)=\sum_{n=0}^{\infty} a_{n} X^{n}
$$

Set

$$
g(X)=g(G, S, X)=\sum_{n=0}^{\infty} b_{n} X^{n} .
$$

The growth series of a pair $(G, S)$ tends to be an interesting power series. For example, it is often a rational function. Rationality questions are considered in almost all of the references. Assume that $G$ has almost finite cohomological dimension, so that the Euler characteristic $\chi(G)$ is defined (see [7]). In this case, computations show that $f(1)$ is closely related to $\chi(G)$. Specifically, if $f$ is meromorphic at 1 and $\chi(G)$ is defined, then until now it has turned out that

$$
f(1)=1 / \chi(G)
$$

in all examples for which both terms were known (see [8 and 9, p. 126]). The purpose of this note is to provide counterexamples to this. After I found the counterexamples given below, Floyd and Plotnick [5] discovered an interesting expression for the difference $f(1)-1 / \chi(G)$ in the case of geometric generating sets of Fuchsian groups. This expression is usually 0 , but they produce examples for which it is not 0. This gives more examples for which the equality in (1) fails.

The counterexamples given below involve considering the relationship between $f(G, S, X)$ and $f\left(G, S_{m}, X\right)$, where $S_{m}=B(m)$ for some $m \geq 1$. It is clear that

Received by the editors February 21, 1986 and, in revised form, September 20, 1986.

1980 Mathematics Subject (lassification (1985 Revision). Primary 20F05, $20 J 05$. 
every such set $S_{m}$ generates $G$, giving rise to $B_{m}(n), a_{m, n}, b_{m, n}, f_{m}(X)$, and $g_{m}(X)$. Clearly, $B_{m}(n)=B(m n)$. Thus

$$
g_{m}(X)=\sum_{n=0}^{\infty} b_{m, n} X^{n}=\sum_{n=0}^{\infty} b_{m n} X^{n}
$$

Now let $\zeta=e^{2 \pi i / m}$. Then

$$
\frac{1}{m} \sum_{k=0}^{m-1} g\left(\varsigma^{k} X\right)=\sum_{n=0}^{\infty} b_{m n} X^{m n}=g_{m}\left(X^{m}\right)
$$

because upon collecting terms in the first expression, the coefficient of $X^{i}$ is 0 if $i \not \equiv 0 \bmod m$ and is $b_{i}$ if $i \equiv 0 \bmod m$. Since $f(X)=(1-X) g(X)$,

$$
f_{m}\left(X^{m}\right)=\left(1-X^{m}\right) g_{m}\left(X^{m}\right)=\frac{1}{m} \sum_{k=0}^{m-1} \frac{1-X^{m}}{1-\varsigma^{k} X}\left(1-\varsigma^{k} X\right) g\left(\varsigma^{k} X\right)
$$

and so

$$
f_{m}\left(X^{m}\right)=\frac{1}{m} \sum_{k=0}^{m-1} \frac{1-X^{m}}{1-\varsigma^{k} X} f\left(\varsigma^{k} X\right) .
$$

Using this to compute $f_{m}(1)$, it follows that if $f(X)$ has no poles at roots of unity, then the term for $k=0$ gives $f(1)$ and all other terms are 0 . However, if $f(X)$ has poles at roots of unity, then it may happen that $f_{m}(1) \neq f(1)$.

For example, start with the group $\mathbf{Z}$ and generating set $\{2,3\}$. It is easy to see that

$$
\begin{aligned}
f(\mathbf{Z},\{2,3\}, X) & =1+4 X+8 X^{2}+6 X^{3}+6 X^{4}+6 X^{5}+6 X^{6}+\cdots \\
& =\frac{1+3 X+4 X^{2}-2 X^{3}}{1-X} .
\end{aligned}
$$

(This appears in both [4 and 9].) Take two copies $\left(G_{1}, S_{1}\right) \cong\left(G_{2}, S_{2}\right) \cong(\mathbf{Z},\{2,3\})$ of this group and generating set and form their free product $(G, S)=$ $\left(G_{1} * G_{2}, S_{1} \cup S_{2}\right)$. For a general free product, one can easily show that

$$
\frac{1}{f(G, S, X)}-1=\frac{1}{f\left(G_{1}, S_{1}, X\right)}-1+\frac{1}{f\left(G_{2}, S_{2}, X\right)}-1
$$

so that

$$
\frac{1}{f(X)}=\frac{2(1-X)}{1+3 X+4 X^{2}-2 X^{3}}-1, \quad f(X)=\frac{1+3 X+4 X^{2}-2 X^{3}}{(1+X)\left(1-6 X+2 X^{2}\right)} .
$$

Now take $m=2$ in the previous notation, namely, view $G$ as generated by the 2-ball for the generating set $S$. For the new generating function $f_{2}(X)$,

$$
\begin{aligned}
f_{2}\left(X^{2}\right) & =\frac{1}{2}\left(\frac{1+3 X+4 X^{2}-2 X^{3}}{1-6 X+2 X^{2}}+\frac{1-3 X+4 X^{2}+2 X^{3}}{1+6 X+2 X^{2}}\right) \\
& =\frac{1+24 X^{2}-4 X^{4}}{1-32 X^{2}+4 X^{4}}
\end{aligned}
$$

and so

$$
f_{2}(X)=\frac{1+24 X-4 X^{2}}{1-32 X+4 X^{2}}
$$


However,

$$
f_{2}(1)=-\frac{7}{9} \neq-1=\frac{1}{\chi(G)} .
$$

Thus this gives a generating set for the free group on two generators for which (1) is false.

Here is an example of a different sort. The growth series for the compact hyperbolic Coxeter groups on three generators using the standard generating sets are determined in [2] (see also [4]). The growth series for the $(2,5,6)$-compact hyperbolic Coxeter group with standard generating set is

$$
f(X)=\frac{(X+1)^{2}\left(X^{4}+X^{2}+1\right)\left(X^{4}+X^{3}+X^{2}+X+1\right)}{\left(X^{2}+1\right)\left(X^{8}-X^{6}-X^{5}-X^{3}-X^{2}+1\right)} .
$$

As Serre showed in [7], equation (1) holds in this case. However, $f(X)$ has poles at $\pm \sqrt{-1}$, which suggests that (1) fails for this group using the 4-ball of the standard generating set as generating set. A routine computation shows that this is indeed the case. There are presumably many such examples.

\section{REFERENCES}

1. M. Benson, Growth series of finite extensions of $\mathbf{Z}^{n}$ are rational, Invent. Math. 73 (1983), 251-269.

2. N. Bourbaki, Groupes et algèbres de Lie, Chapitres 4, 5, et 6, Hermann, Paris, 1968.

3. J. Cannon, The combinatorial structure of cocompact discrete hyperbolic groups, Geom. Dedicata 16 (1984), 123-148.

4. - The growth of the closed surface groups and the compact hyperbolic Coxeter groups, preprint.

5. W. Floyd and S. Plotnick, Growth functions on Fuchsian groups and the Euler characteristic, Invent. Math. (to appear).

6. M. Grayson, Geometry and growth in three dimensions, Ph.D. Thesis, Princeton Univ., 1983.

7. J.-P. Serre, Cohomologie des groupes discrets, Prospects in Math., Ann. of Math. Studies, no. 70, Princeton, Univ. Press, Princeton, N. J., 1971.

8. N. Smythe, Growth functions and Euler series, Invent. Math. 77 (1984), 517-531.

9. P. Wagreich, The growth function of a discrete group (Proc. Conf. on Group Actions and Vector Fields), Lecture Notes in Math., vol. 956, Springer-Verlag, Berlin and New York, 1982, pp. 125-144.

Department of MAthematics, EAstern Michigan University, Ypsilanti, MICHIGAN 48197 